Article

\title{
New Design of Phase-Shifted Full Bridge Power Converter for Photovoltaic Application
}

\author{
Wasan Phetphimoon and Krischonme Bhumkittipich * (1) \\ Department of Electrical Engineering, Rajamangala University of Technology Thanyaburi, Pathum Thani \\ 12110, Thailand; wasan_p@mail.rmutt.ac.th \\ *Correspondence: krischonme.b@en.rmutt.ac.th; Tel.: +66-02-549-3571
}

\begin{abstract}
This paper presents the design of a high frequency zero voltage switching (ZVS) full-bridge converter with a phase-shifted driving signal for photovoltaic applications. The resonant power converter can provide high-power capacity under high-frequency operation. The proposed power converter can also reduce the size of the transformer under the same power rating. The high-frequency transformer was developed by using the resonant and switching frequencies of the power converter to reduce the switching loss and to improve the system efficiency. Phase-shifted modulation was selected to drive the switches of a full-bridge power converter based on the switching loss minimization method. The desired output voltage was controlled using a closed-loop controller under a loop gain stability margin. The simulation results showed that the output voltage can be controlled to the desired constant when the input voltage changes from 30 $V_{D C}$ to $60 \mathrm{VDC}$. The desired output voltage of power converter is constant at $400 \mathrm{VDC}$. The power converter can transfer the DC supply to a $220 \mathrm{~V}_{\mathrm{AC}}$ household via grid-connected inverter. Therefore, the proposed study showed the effectiveness of the phase-shift ZVS full-bridge power converter with high-frequency transformer. This power converter can control the operation of the desired voltage system and has a small sizing of power converter system, low switching loss, and high system efficiency.
\end{abstract}

Keywords: phase-shifted full-bridge, resonant converter, photovoltaics, zero voltage switching, power loss.

\section{Introduction}

Photovoltaic systems are renewable energy systems in which solar energy is transformed into electricity using semiconductor materials. Photovoltaic systems can replace electric power plants because of fossil fuel usage, energy availability, environmental pollution due to carbon dioxide emissions, and rapid fossil fuel consumption. Integrating solar energy sources into existing energy infrastructure is derived by the general installation of distributed generation for increasing power system stability. Photovoltaic systems use electronic equipment as power converters to convert the solar panel output to the desired voltage. Therefore, an important part of photovoltaic systems is a power converter that can draw the solar energy power to power grid systems. The control indices of a photovoltaic system are value, performance and power loss in the future [1-3]. To solve these indices, the photovoltaic system can increase the power capacity using modern power converter technology, increase the high-power capacity of transformers, and reduce the size of the transformer using high-frequency operation. For the above design purpose, this paper has selected to use a high-frequency high power converter that can control the desired voltage rapidly and can also isolate the ground system from the electrical system. 
A high-power converter uses soft switching operations, namely, zero voltage switching (ZVS), that reduces the switching loss of the power converter during on and off switch operation. The resonant DC-DC power converter is driven by the phase-shifted PWM modulation technique [4-5]. Therefore, this power converter can reduce switching power loss and increase overall conversion efficiency [6-7]. For the above reason, this article will propose new control methods for power converter to improve the system performance. The two parallel half-bridge converters and an initial starter inductor are selected. Therefore, this paper presents the control for DC-DC power converters. It provides a path for power flow and a wider load range for lightweight conditions without additional power circuits. In addition, the proposed controller method combines drive logic for better power converter performance. The mathematical models explain the principles and advantages of the proposed control algorithm methods, including linear phase-shifted voltage and phase-to-weight ZVS operation. Fig.1 shows the proposed power converter for a photovoltaic system. This power converter topology consists of a full bridge converter, rectifier circuit, high-frequency transformer and grid-connected inverter with a boost converter. The converter can operate in bidirectional power flow mode and can turn-on/turn-off in the soft switched operation mode for a wider load range.

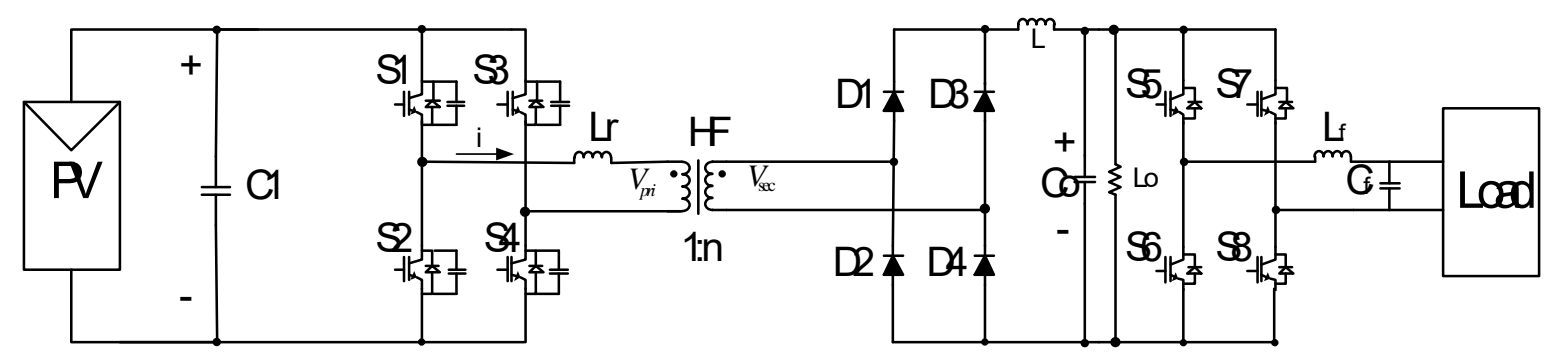

Figure 1. The full-bridge power converter for photovoltaic systems.

\section{Analysis of The Phase-Shifted Full Bridge Power Converter}

The phase-shift ZVS full-bridge power converter is the DC-DC power converter with three cascade parts for power conversion. The first part is a DC to AC phase-shift full-bridge converter with high frequency primary transformers sides, the second part is an $\mathrm{AC}$ to $\mathrm{AC}$ high frequency secondary transformer sides, and the third part is an AC to DC high-frequency rectifier. In the topology of the full bridge phase shift ZVS converter, there are four switching elements, such as a full-bridge converter [8]. The switches of $S_{1}$ and $S_{2}$ are turned-on and turned-off complementarily with a $48 \%$ duty cycle. Conversely, the opposite switches $S_{3}, S_{4}$ are turned-on with a different strategy. The switches $S_{3}$ and $S_{4}$ are phase-shifted according to the desired control signal.

The inductor energy for achieving ZVS can be calculated as equation (1).

$$
E=\frac{1}{2} \cdot L_{r} \cdot I_{2}^{2} \geq \frac{3}{4} \cdot C_{m o s} \cdot V_{i n}{ }^{2}+\frac{1}{2} \cdot C_{T R} \cdot V_{i n}^{2}
$$

where $I_{2}$ is the current flowing through the primary side when $S_{2}$ is stopped, $V_{\text {in }}$ is the input voltage of the circuit and $C_{o}$ is the capacity of the transformer coil. The transformer factor of $3 / 4$ is the double power stored in a nonlinear drain. The capacity varies according to the square root of 
the input voltage [9]. The resonance between the values of $L_{r}, C_{m o s}$ and $C_{T R}$ lets the sinusoidal voltage pass the maximum capacity at one-fourth of the resonant frequency range. It can set dead time between $S_{3}$ and $S_{4}$ by setting the value of $\delta t_{\max }$ in order to be sure that there is sufficient charging time and discharging the charge at the specified time to ensure that ZVS while connecting the highest possible load can be determined by the following equation (2).

$$
\delta t_{\max }=\frac{T}{4}=\frac{\pi}{2} \sqrt{L_{r} \cdot C}
$$

where $C=C_{m o s}+C_{o}$.

The interval of zero switching conditions of $S_{3}$ and $S_{4}$ will vary with the load level of the converter. Because the load current is low when closed, $S_{3}$ and $S_{4}$ may not be sufficient to activate for parallel protection signal, for switches $S_{1}$ and $S_{2}$, and the ZVS has a different mechanism. Before $S_{1}$ is turned off, the main current is reaching the maximum value. When $S_{1}$ is turned off, the available power to charge the output capacity of $S_{1}$ and discharge the output capacity of $S_{2}$ is the energy stored in $L_{r}$. This inductive filter output is provided because the filter inductor does not rotate through the regulator until the second voltage is reduced to zero. The energy inductance of the filter is large when compared to the energy stored in the switch capacitor. The current switching method implementation can be estimated by charging in the linear form with constant current. Therefore, the $\delta t_{1}$ dead time which required during turn-on and turn-off of the $S_{1}$ and $S_{2}$ can be obtained in equation (3).

$$
\delta t_{1} \cdot I_{p}=4 \cdot C_{m o s} \cdot V_{i n}
$$

The 4. $C_{m o s} \cdot V_{\text {in }}$ corresponds to the double charging stored in nonlinear output. The capacity of electronic switches and $I_{p}$ is the maximum current in the inductive filter output. The reflection of the main dead time can be calculated as the lowest value selected to get the ZVS value. If the current is reduced, the ZVS value cannot be controlled. For the ZVS occurrence of the $S_{1}$ and $S_{2}$ operating range can be achieved even at the light load due to the fact that $\mathrm{D}_{1}$ and $\mathrm{D}_{3}$ can be opened by the energy stored in the output filter inductor. However, ZVS for $S_{2}$ and $S_{4}$ can only be used for load currents that are higher than the critical values shown in the equation (4). 


$$
I_{C R I T}=\sqrt{\frac{2}{L_{r}} \cdot\left(\frac{4}{3} \cdot C_{m o s} \cdot V_{i n}^{2}+\frac{1}{2} \cdot C_{t r} \cdot V_{i n}^{2}\right)}
$$

The current through $L_{r}$ at $t_{2}$ can calculate such values from the equation (5).

$$
I_{2}=\frac{N_{s}}{N_{p}} \cdot\left(I_{\text {load }}+\frac{\Delta I}{2}-\frac{V_{\text {out }}}{L_{r}+L_{m}}(1-D) \frac{T}{2}\right)
$$

Finally, the value of the ZVS is able to receive the load current at $I_{2}>I_{C R I T}$, which is shown in equation (6).

$$
I_{\text {load }} \geq \frac{N_{p}}{N_{s}} \cdot I_{C R I T}-\frac{\Delta I}{2}+\frac{V_{\text {out }}}{L_{r}+L_{F}} \cdot(1-D) \frac{T}{2}
$$

\subsection{Operation State of the DC-DC Power Converter}

The power converter equivalent circuits directly from the full bridge DC-DC power converter circuit are shown in Figure 2. The switch can be partitioned into two sets of state-based sub-circuits, switches, and shortcuts for the status of the switch: $0 \leq \mathrm{t} \leq \mathrm{dT}_{\mathrm{s}}, \mathrm{dT}_{\mathrm{s}} \leq \mathrm{t} \leq, \mathrm{T}_{\mathrm{s}}$. The induced current and the capacitor voltage were chosen as a state variable of output voltage [10].

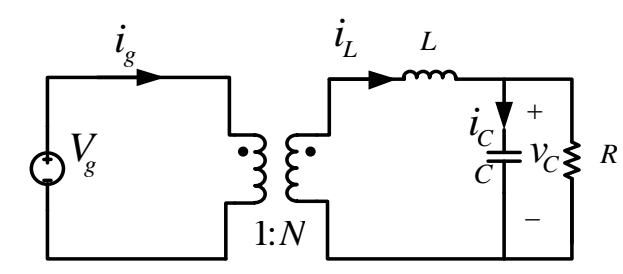

Figure 2. The equivalent circuit of DC-DC power converter

In the $0 \leq \mathrm{t} \leq \mathrm{dTs}$ period, $S_{1}, S_{4}$ or $S_{2}, S_{3}$ or performing a, Fig. 2 is the equivalent circuit of the DC-DC power converter and its equation is shown in equation (7)

$$
\left\{\begin{array}{l}
L \frac{d i_{L}}{d t}=\frac{V_{g}}{n}-V_{C} \\
C \frac{d v_{C}}{d t}=i_{L}-\frac{v_{C}}{R}
\end{array}\right.
$$

In the dTs $\leq \mathrm{t} \leq \mathrm{T}$ period, $S_{1}, S_{4}$ or $S_{2}, S_{3}$ are turned-off. Fig. 2 (a) shows the equivalent circuit, and its equation is as expressed in equation (8).

$$
\left\{\begin{array}{l}
L \frac{d i_{L}}{d t}=-V_{C} \\
C \frac{d v_{C}}{d t}=i_{L}-\frac{v_{C}}{R}
\end{array}\right.
$$


Then, for equations $(7)$ and $(8)$, the average value is $(7) \times d+(8) \times(1-d)$, the duty cycle is the $d$ value in the equation (9).

$$
\left\{\begin{array}{l}
L \frac{d i_{L}}{d t}=2 d\left(\frac{V_{g}}{n}-\mathrm{v}_{C}\right)+2 \mathrm{~d} \frac{-v_{C}}{T_{S}} \\
C \frac{d v_{C}}{d t}=2 d\left(\mathrm{i}_{L}-\frac{v_{C}}{R}\right)+2 d\left(\mathrm{i}_{L}-\frac{v_{C}}{R}\right)
\end{array}\right.
$$

\subsection{The Small-Signal Model of a Full Bridge DC-DC Power Converter}

Figure 3 shows a small signal circuit for a full bridge converter that can be obtained with a PWM signal converter. The duty cycle is controlled by the values of inductor voltage and current input [11]. The effective duty cycle can be expressed in equation (10).

$$
d_{e f f}=D_{e f f}+\hat{d}_{e f f}
$$

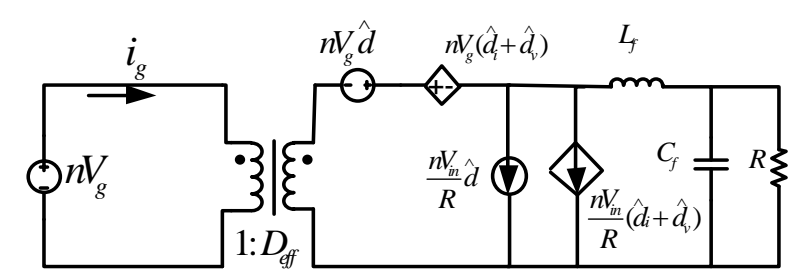

Figure 3. The small-signal model of the proposed power converter.

An effective cycle time depends not only on the duty cycle. But it depends on the primary voltage, inductor filter and the drop voltage of leakage inductor. The effective duty cycle of power converter is constant and small duty cycle changing, due to the inductor current and input voltage, can be shown as given in equation (11).

$$
\hat{d}_{\text {eff }}=\hat{d}+\hat{d}_{i}+\hat{d}_{v}
$$

$\hat{d}_{i}$ and $\hat{d}_{v}$ are the small change of the duty cycle due to the filter inductor current and the input voltage changing as the equation (12) and (13)

$$
\begin{aligned}
& \hat{d}_{i}=\frac{4 n L_{r} f_{s}}{V_{i n}} \hat{i}_{L} \\
& \hat{d}_{v}=\frac{4 n L_{r} f_{s}}{V_{i n}} \hat{V}_{i n}
\end{aligned}
$$

Transfer function from equivalent circuit of Fig. 3, as shown in equation (14). 


$$
G(\mathrm{~s})=\frac{\hat{v_{o}}}{\hat{d}}=\frac{n V_{i n}}{L C} \frac{1}{s^{2}+s\left(\frac{L}{R}+R_{d} C\right)+\left(\frac{R_{d}}{R L C}+\frac{1}{L C}\right)}
$$

\subsection{Steady State Analysis of Phase-Shift Full-Bridge Power Converter}

The reducing the size and weight of magnetic components is desirable as is increasing the switching frequency for DC-DC power converters. When conventional PWM converters operate at high frequencies, the circuit performance will also decrease. Increased switching loss in high power applications must have insulting or other protection methods that cause significant losses and reduce efficiency.

When the operation full bridge converter uses the shift phase control signal, there are two functions. The first step is to convert DC to AC by full bridge converter circuits, connected with a high frequency transformer, and the second step is an AC to DC by high frequency rectifier circuits with a low-pass filter. The full bridge converter has four switches, similar to the conventional full bridge converter shown in Fig. 1. The proposed control for switching $S_{1}-S_{4}$ is a phase-shifted PWM that differs from conventional control. The leg-pair switches are turned on complementarily with $48 \%$ duty cycle excluding the short dead time. The driving signals are shown in Fig. 4 (b) where the second control signal has shifted the phase. The operation in the switch mode is zero, resulting in the current and voltage generated by the switch operation 1-4 as shown in Figure 4 (c)

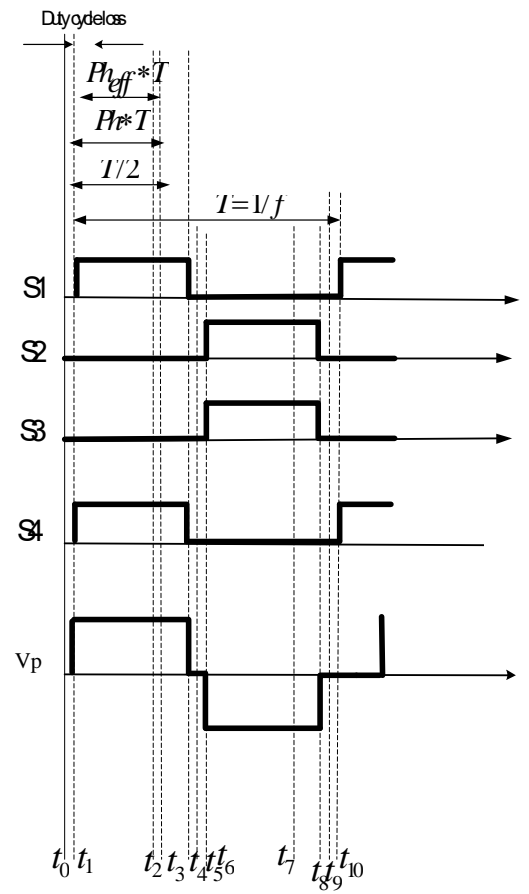

(a)

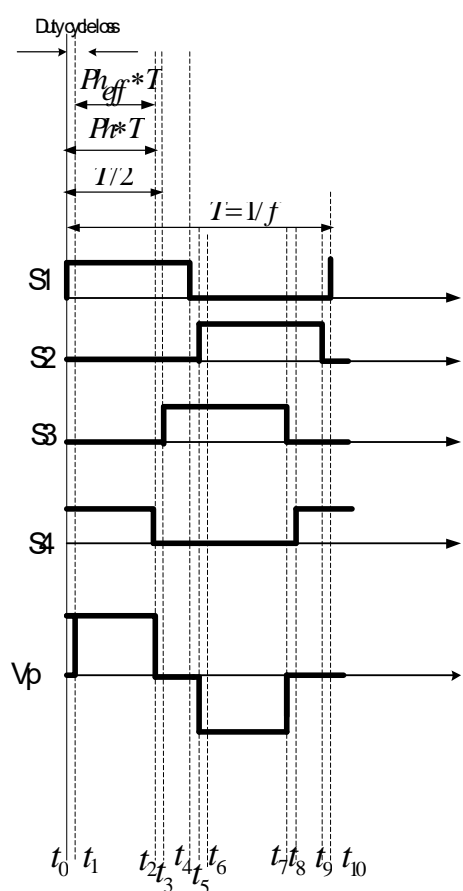

(b)

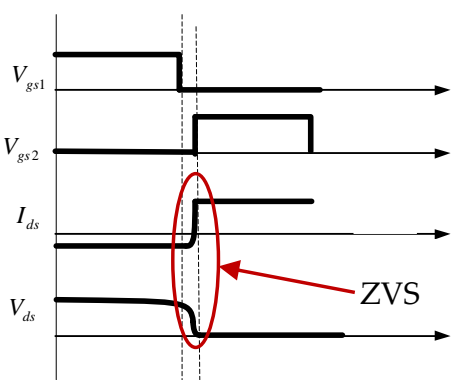

(c)

Figure 4. (a) Conventional full-bridge PWM Converter (b) Principle of operation for the full bridge phase- shifted power converter (c) The circuit for the ZVS mode of switch [12]. 
Figure 4 shows the driving signals of $S_{1}$ to $S_{4}$, the primary voltage side of transformer, $V_{P}$ and the secondary voltage side of transformer Vs. The operation state can be explained by each mode as follows [13-15]:

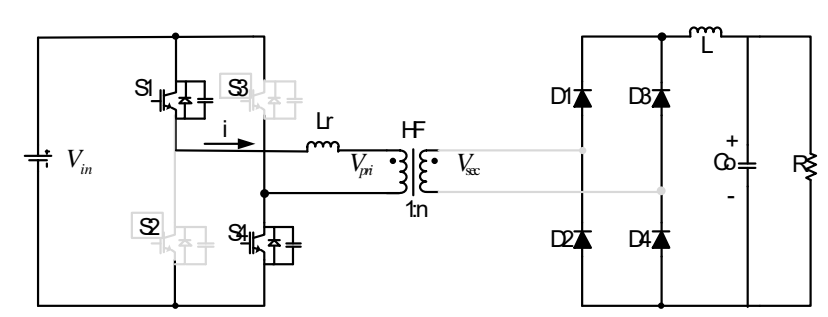

Figure 5. Mode 1

Mode 1: Loss of duty cycle

In mode $t=t_{0}$, the switches $S_{1}$ and $S_{4}$ are on; switch $S_{1}$ is turned on with ZVS after ending mode $t_{0}$. The secondary voltage side of transformer $V_{s}$ remained zero until the current primary side of the transformer reverses to the positive direction and increased to reach the reflected output inductor current $\left(n I_{L o}\right)$ in mode loss of duty cycle $t=t_{1}$.

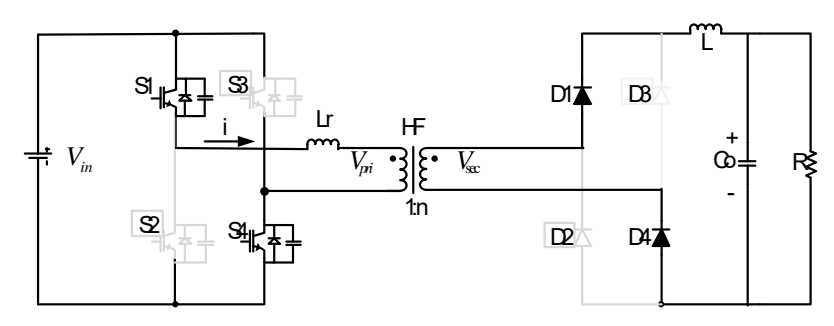

Figure 6. Mode 2

Mode 2: Power transfer mode

In mode $t=t_{1}$, the secondary voltage of the transformer is equal to $n V i n$, the $D_{1} D_{4}$ rectifier diodes are forward biased and transfer energy from source to load. The first operation mode of the phase shift $t_{1}-t_{2}$, the current across the output filters began to increase and the primary current of the transformer $I_{p}$ then equal to the output inductor current that reflects $\left(n I_{L o}\right)$.

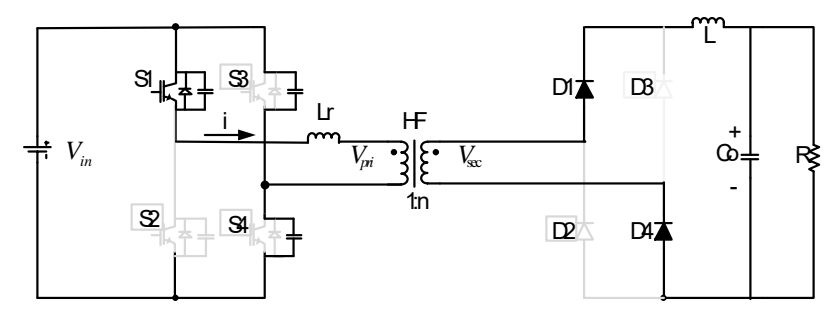

Figure 7. Mode 3

Mode 3: Zero voltage for the $S_{3}$ switch 
At In mode $t=t_{2}$, the $S_{4}$ switch is off, and the primary current of the transformer charges the $C_{4} S_{4}$ switch and discharges the switch of $S_{3}$ when charging capacity $C_{3}$ is fully released. From $V_{i n}$ to 0 , the $D_{3}$ diode will start to obtain zero voltage for the $S_{3}$ switch. This will reduce the damage to the switch. In this mode, the secondary voltages of the transformer turn into zero. The high frequency rectifier diode $D_{1}$ and diode $D_{4}$ will conduct the electricity.

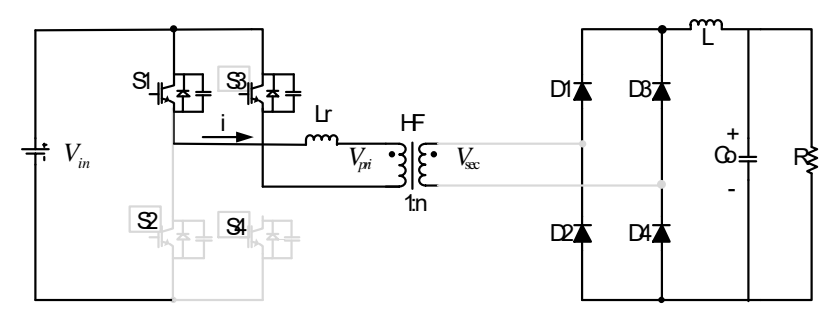

Figure 8. Mode 4

\section{Mode 4: Freewheel though switch mode}

In mode $t=t_{3}$, the $S_{3}$ switch with the ZVS and primary current of the high frequency transformer $I_{P}$ is turned-on and freewheeled though $S_{1}$ and $S_{3}$. The secondary voltage of the transformer $V_{S}$ in the state of zero and the output voltage is released by the load.

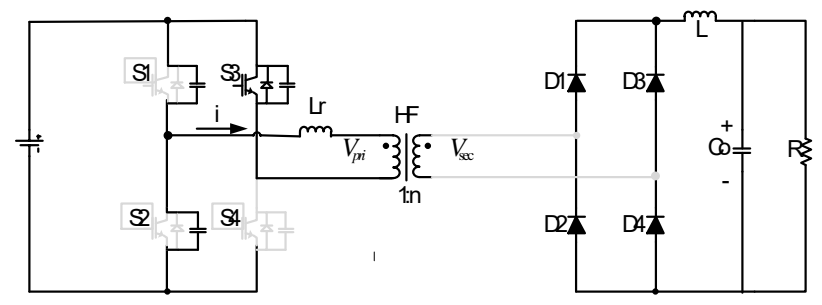

Figure 9. Mode 5

Mode 5: Zero voltage for the $S_{2}$ switch

In mode $t=t_{4}$, while the switch $S_{1}$ is in the down state and the primary current of the transformer will be in the charging state $C_{1}$ connected with $S_{1}$ and then discharge $C_{2}$ that is connected to the switch $S_{2}$. When the capacitance $C_{2}$ was completely released from $V_{\text {in }}$ to 0 , diode $D_{2}$, it will be in mode is zero voltage due to the switch for switch $S_{2}$. The secondary voltage of the transformer remains zero voltage during the mode $t_{4}-t_{5}$.

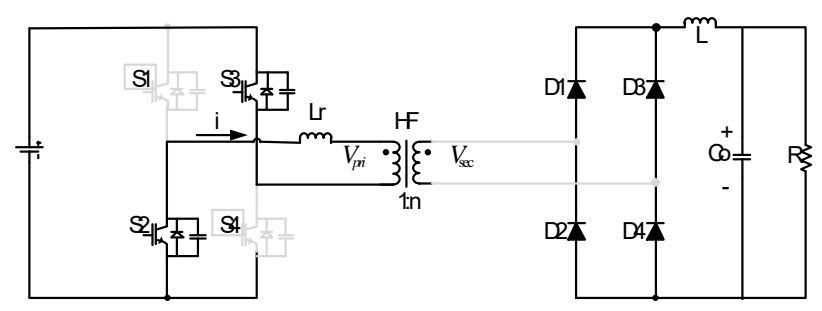


Figure 10. Mode 6

Mode 6: Loss of duty cycle

In mode $t=t_{5}$, switch $S_{2}$ Enable with ZVS after the end of mode $t_{4}$. The secondary voltage of the transformer $V_{s}$, still at zero voltage until the primary current of the transformer $I_{P}$ reverses its direction and starts to increase the induction of the output current reflected $n I_{L o}$ at period $t=t_{6}$. The primary current of the transformer will increase according to the slope $V_{i n} / L_{r}$ as the $V_{\text {in }}$ which ignored the induced leaking inductor $L_{r}$ and lack of power transfer to the output.

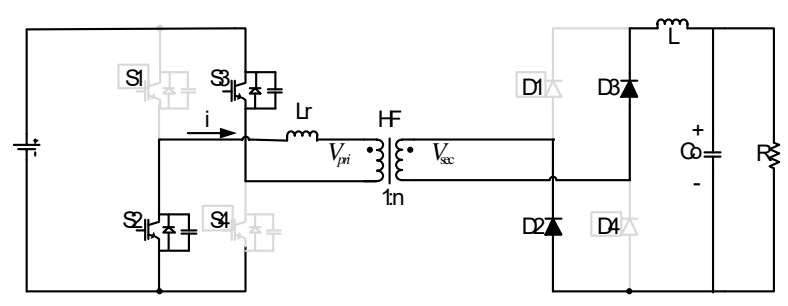

Figure 11. Mode 7

\section{Mode 7: Power transfer mode}

In mode $t=t_{6}$, the secondary voltage side of the transformer $V_{s}$ equals $n V_{i n}$. The high frequency rectifier diodes $D_{2}$ and diodes $D_{3}$ are the period in which the forward biased diode and transfer energy to charge the inductor to pass power output. The first operation mode of the phase shifts again the working range of mode $t_{6}$. Cross-inductor output currents begin to increase, and the primary current of the transformer $I_{P}$ flows in the negative direction equal to the electric current that is reflected $n I_{L o}$.

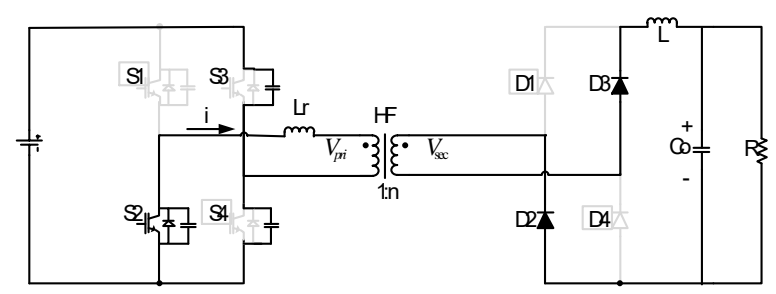

Figure 12. Mode 8

Mode 8: Zero voltage for the $S_{2}$ switch

In mode $t=t_{7}$, while the switch is in the down state and the primary current of the high frequency transformer will be in the charging state $C_{3}$ connected with $S_{3}$ and then discharge $C_{4}$ that is connected to the switch $S_{4}$. When the capacitance $C_{4}$ was completely released from $V_{i n}$ to 0 , its device diode $D_{4}$ was in the mode of zero voltage due to the switch $S_{4}$. In this mode, the secondary voltage of the high frequency transformer will become zero and the high frequency rectifier diode will carry the current. 


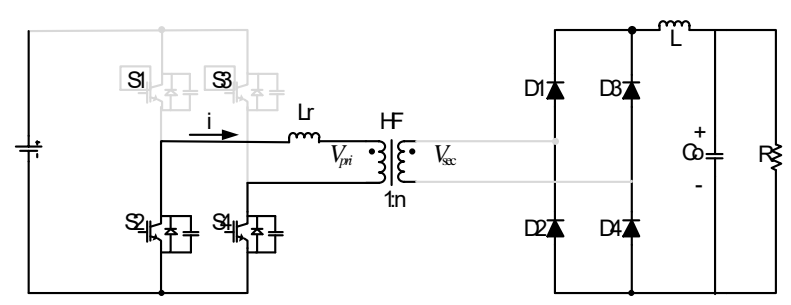

Figure 13. Mode 9

Mode 9: Freewheel though switch mode

In mode $t=t_{8}$, switch $S_{4}$ with the ZVS and primary current of the high frequency transformer $I_{P}$ is turned on and freewheeled though $S_{2}$ and $S_{4}$. The secondary voltage of the high frequency transformer $V_{S}$ in the state of zero and the output voltage is released by the load.

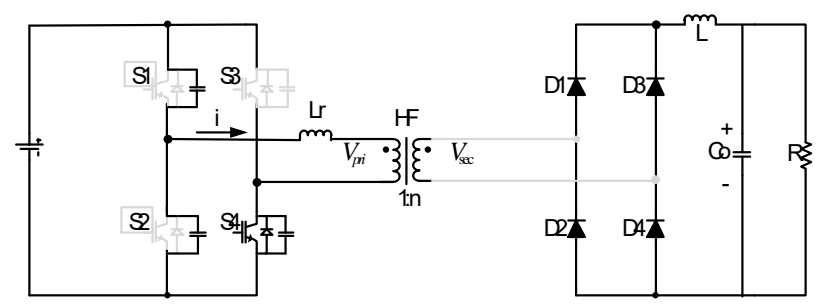

Figure 14. Mode 10

Mode 10: Zero voltage for the $S_{2}$ switch

In mode $t=t_{9}$, switch $S_{2}$ while the switch was in the down state and the primary current of the high frequency transformer was in the charging state $C_{2}$ connected with $S_{2}$ and then discharge $C_{1}$ that was connected to the switch $S_{1}$. When the capacitance $C_{1}$ was completely released from $V_{\text {in }}$ to 0 , its device diode $D_{1}$ was in the mode of zero voltage due to the switch $S_{1}$ solving problems of loss in switch devices. In this part, the secondary voltage of the high frequency transformer returned to zero, and in the rectifier circuit $D_{2}$, and $D_{3}$ was in current condition.

\section{High-frequency Transformer}

High frequency transformers are designed to support integration with electronic switches in the form of high frequency signals. And they are designed to obtain the output voltage that is maintained between the lowest input voltage when the maximum load is connected. The duty cycle is an important parameter in controlling the operation of the system to transfer the energy of the transformer from the primary side to the secondary side. The offset time of each working period is half-worked alternately in order to reverse the primary side of the system and to allow the current to flow to the current circuit on the secondary side of the high frequency transformer. As a result, having to consider the loss of work cycles when calculating the rotation ratio. Otherwise, the converter may have a high loss value [16]. Especially during the control period under extreme load conditions, especially if it exists. For higher leakage induction values [17], the voltage is shown in the equation (15). 


$$
\frac{V_{o}}{V_{i n}}=\frac{N_{s}}{N_{p}} \cdot p h-I_{o} \cdot\left(\frac{N_{s}}{N_{p}}\right) \cdot \frac{L_{r}}{V_{i n}} \cdot f
$$

For the energy loss from transformer ratio, without the cupper loss, can be can be calculated by this equation (16).

$$
\text { pheff }=\frac{V_{o}}{V_{\text {in }}} \cdot \frac{N_{p}}{N_{s}}
$$

The principle of choosing the size and shape of the transformer will mainly consider the efficiency and increasing temperature. Some transformer design experiences, along with a few repetitions, need to choose the most appropriate axis with a balanced core and loss of coil.

For the loss of an acceptable axis, determine the maximum magnetic flux limit at 0.1 Tesla. Therefore, the number of primary windings of the transformer can be calculated.

$$
\begin{aligned}
& N_{p}=\frac{V_{i n} \cdot p h_{e f f}}{2 \cdot B_{\text {max }} \cdot A_{c} \cdot f} \\
& B_{\max }=\frac{V_{i n} \cdot p h_{e f f}}{2 \cdot N_{p} \cdot A_{c} \cdot f}
\end{aligned}
$$

The ripple of inductor current can be calculated from the equation below.

$$
\Delta I_{L 1}=\% \text { Ripple } \cdot \frac{I_{o}}{2}
$$

The inductance of the L1 filter and the maximum current are determined according to the induced ripple current [18].

$$
L_{1}=\frac{1}{\Delta I_{L 1}} \cdot V_{o}\left(1-p h_{e f f}\right) \cdot T
$$

Output of the capacitor ripple current is a function other than the phase shift of the power conversion circuit. Current rectifier can completely reduce the current ripple in capacity in the case of Pheff $=0.48$. Current ripple output capacitor, RMS current and ESR loss can be calculated by the following equation.

$$
\begin{aligned}
& \Delta I_{C o}=\frac{V_{o}}{L_{1}} \cdot T \cdot\left(1-2 \cdot p h_{\text {eff }}\right) \\
& I_{\text {Co.rms }}=\sqrt{\frac{1}{12} \cdot \Delta I_{\text {Co }}^{2}} \\
& P_{\text {Co }}=I_{\text {Co.rms }^{2}} \cdot E S R_{\text {out }}
\end{aligned}
$$


The ripple voltage of the capacitor value can be calculated from the equation

$$
\Delta V_{C o}=\frac{V_{o} \cdot\left(1-2 \cdot p h_{e f f}\right) \cdot T^{2}}{16 \cdot L_{f} \cdot C_{o}}
$$

The capacitor value used to filter the output voltage can be calculated as

$$
C_{o}=\frac{V_{o} \cdot\left(1-2 \cdot p h_{e f f}\right) \cdot T^{2}}{16 \cdot L_{m} \cdot \Delta V_{C o}}
$$

\section{Design of Phase-Shifted Full-Bridge Power Converter}

This section discusses the design of power converters for photovoltaic systems. The system component in the first part is the full bridge phase-shift ZVS converter that is connected to the high frequency transformer and in the second part is the high frequency rectifier circuit by parameters as shown in the table 1.

Table 1. Parameters of designed power converter

\begin{tabular}{cc}
\hline Items & Value \\
\hline Input voltage $V_{i}$ & $48 \mathrm{~V}$ \\
Input voltage range & $30-60$ \\
Output voltage $V_{o}$ & $400 \mathrm{~V}$ \\
Output voltage ripple $\Delta V_{o}$ & $0.25 \%$ \\
Switching frequency, $f_{s}$ & $50 \mathrm{kHz}$ \\
Maximum duty cycle & 0.48 \\
\hline
\end{tabular}

In order to reduce losses caused by transformer isolation, the ferrite core with low saturation flux density is considered the magnetic core with the highest magnetic flux range $B_{\max }$ of $0.2 \mathrm{~T}$. Area size for core $A_{C}$ of $1.5 \mathrm{~cm}^{2}$, determines the number of coils around the primary side of the high frequency transformer as equation (26).

$$
N_{p}=\frac{V_{\text {in }} \cdot D}{2 \cdot B_{\max } \cdot A_{c} \cdot f} \times 10^{8}
$$

Determine the number of primary windings of the high frequency transformer 6 turns to meet the output voltage requirements and support the voltage across the circuit element. The secondary voltage of the high frequency transformer should be based on the ratio of the output voltage with the maximum duty cycle value. 


$$
V_{\mathrm{sec}} \geq \frac{V_{o}}{D_{\max }}
$$

The duty cycle value is defined as $48 \%$, and the secondary voltage of the high frequency transformer equation is $V_{O}=D_{\text {eff }} \times V_{\text {sec }}$.The number of secondary windings of the high frequency transformer can be calculated from the equation (28):

$$
N_{s}=N_{p} \frac{V_{\text {sec }}}{V_{\text {in }}}=84 \text { Turns }
$$

The inductor value used to filter the voltage is determined by the efficiency input voltage and output voltage and the duty cycle performance value is given by [19]

$$
L_{o}=\frac{\left(\eta \mathrm{V}_{i n}-\mathrm{V}_{o}\right) \mathrm{D}_{e f f}}{\Delta I_{L} f_{s}}
$$

The ripple current $\Delta I_{L}$ for the inductor was set at $10 \%$, and the inductor used for filtering voltage was $0.95 \mathrm{mH}$. The output filter capacitor was determined by selecting the ripple voltage output $\Delta V_{o}=0.25 \%$

$$
\Delta V_{c}=\frac{V_{o}\left(1-\mathrm{D}_{e f f}\right)}{16 f_{s}^{2} C L_{o} f_{s}}
$$

The frequency of achieving the resonance of the ZVS switching between the energy stored in the transformer leakage inductors and capacitor of the MOSFET devices for the four switches as shown in the [20-22]:

$$
f=\frac{1}{2 \pi \sqrt{L_{r} C_{r}}}
$$

The leakage inductance is calculated from:

$$
L_{r}=4 t_{d}^{2} / \pi^{2} C_{r}
$$

\section{Experimental Results}

The full bridge power converter for photovoltaic systems has tested the operation of the circuit, with the simulation in MATLAB Simulink. In the first part of the circuit will be full bridge phase-shift ZVS converter by PWM switching signals $S_{1}-S_{4}$ with the phase shift method to allow the switch of the device to be in ZVS mode and also result in effective voltage control. In the second 
part, it is a high frequency rectifier circuit that converts AC - DC voltage with a diode device $D_{1}-D_{4}$ is presented in Figure 15.
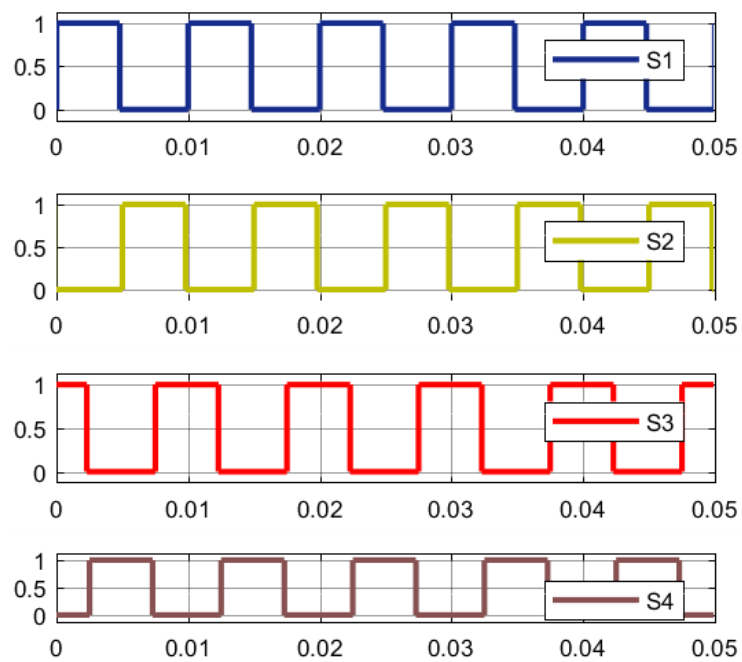

Figure 15. Signal $S_{1}-S_{4}$ full bridge phase shift ZVS dc-dc power converter

PWM switching signals $S_{1}-S_{4}$ with the phase shift method show a simplified circuit of a phase shifted full bridge. The four switches work in a phase shift to control the range of circuits that are connected to the primary side of the high frequency transformer. The duty cycle of the switch signal is $48 \%$. The signal of the switch of $S_{1}$ and $S_{2}$ is divided into half the work time. Similarly, in $S_{3}$ and $S_{4}$ the performance of the phase shift signal is shown in the Fig. 15.

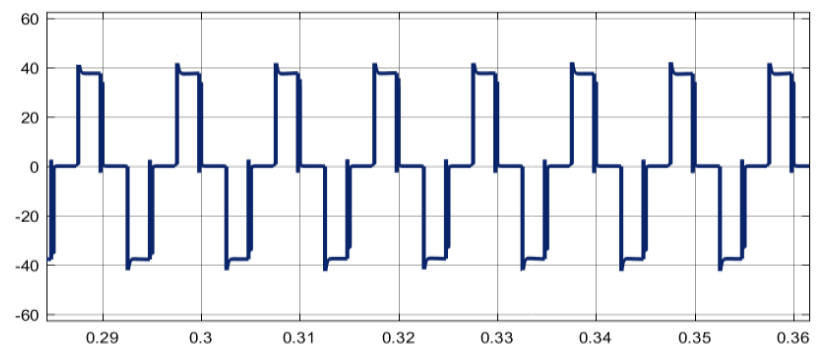

Figure 16. Primary voltage of full bridge phase shift ZVS dc-dc power converter

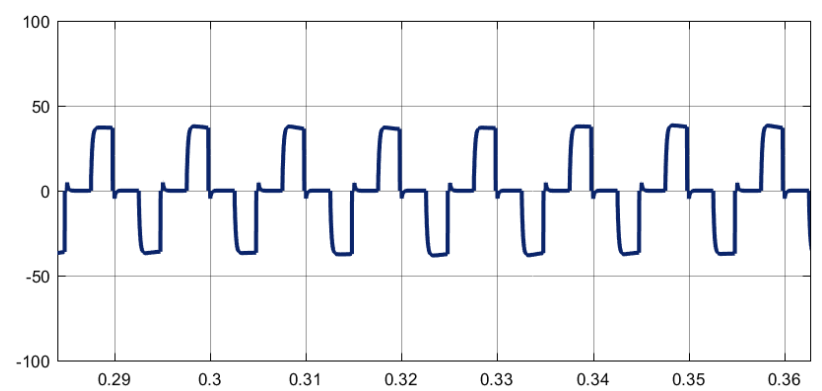

Figure 17. Primary current of full bridge phase shift ZVS dc-dc power converter

This simulation results show the primary voltage and the primary current of the high frequency transformer obtained from the full bridge phase shift ZVS dc-dc converter circuit. The 
PWM waveforms powered by the phase shift method of $S_{1}-S_{4}$ switches according to the conditions described above will be presented in the Fig. 18.

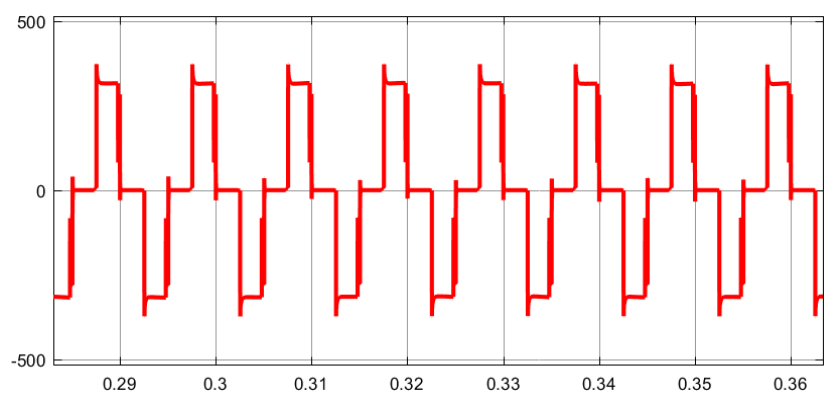

Figure 18. Secondary voltage of full bridge phase shift ZVS dc-dc power converter

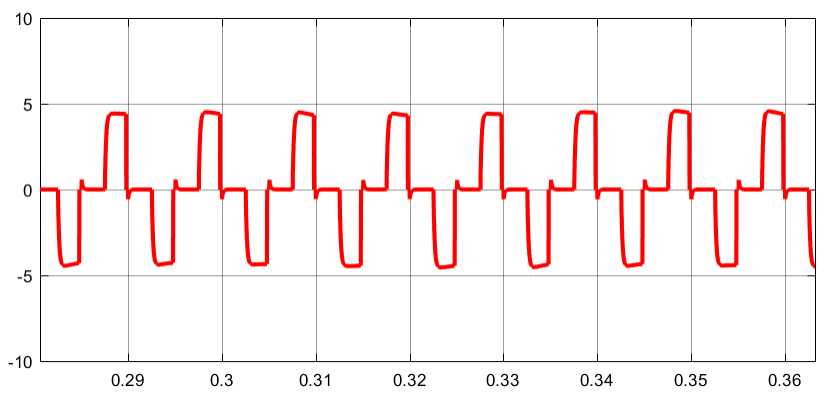

Figure 19. Secondary current of full bridge phase shift ZVS dc-dc power converter

This simulation results show the secondary voltage and secondary current of the high frequency transformer that has the output voltage control as designed as shown in the Figure 18 and 19
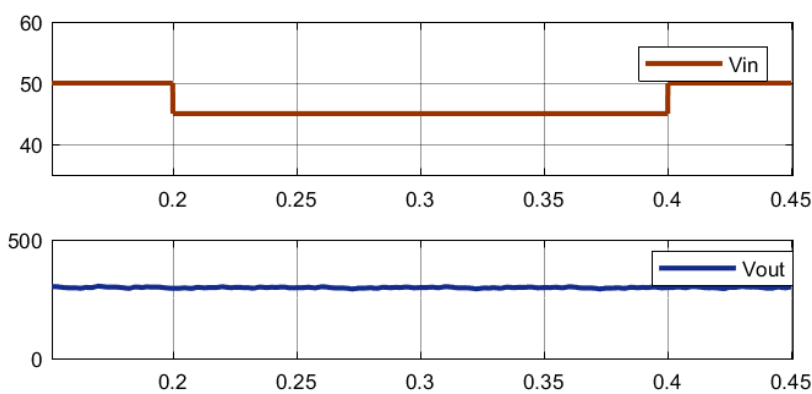

Figure 20. Simulation results of the input voltage when the state changes from full bridge phase shift ZVS dc-dc power converter

Figure 20 shows that when the input voltage changes, the full bridge phase shift ZVS dc-dc converter maintains the rated voltage of $45 \mathrm{~V}-50 \mathrm{~V}$, and the output voltage of the circuit full bridge phase shift ZVS dc-dc converter is able to control $400 \mathrm{~V}$. 

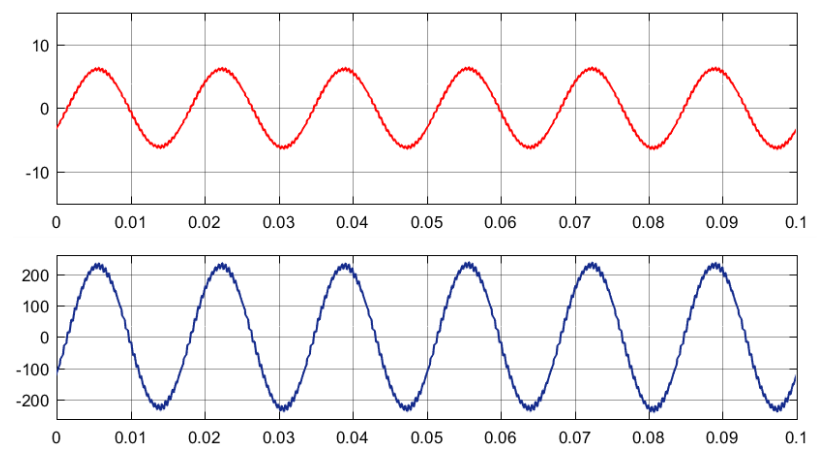

Figure 21. Output voltage and current from the inverter circuit

Figure 21 is a simulation of the inverter circuit that receives electrical power from full bridge phase shift ZVS dc-dc power converter, connected to a load of $1 \mathrm{~kW}$. The inverter circuit will convert the DC voltage to AC voltage in the sinusoidal signal at $220 \mathrm{Vac} 50 \mathrm{~Hz}$. The full bridge phase shift ZVS converter was simulated in MATLAB/Simulink by PWM switching phase-shift signals $S_{1}-S_{4}$ of the full bride dc-dc converters. The phase shift feature allowed the switching device to be the soft-switching that allowed zero voltage switching (ZVS) characteristics, which reduced the size and weight of high frequency transformer. The conventional PWM converters operated at high frequency and power system converters circuits which have an impact system on the converter performance. The phase shift PWM allowed the operation of the device that reduces loss, switching and stress switches for improving energy conversion efficiency.

\section{Conclusions}

The phase-shift ZVS full-bridge power converter operated under the zero-voltage switch principle together with the control signal PWM in the phase-shift technique. The power converter consists of a high frequency full-bridge power converter circuit to increase the DC voltage level at the rated voltage of $40 \mathrm{~V}$ to $370 \mathrm{~V}$, then converts the $\mathrm{DC}$ voltage to $\mathrm{AC}$ voltage via the grid-connected inverter. The output voltage of the inverter circuit is equal to $220 \mathrm{~V}_{\mathrm{AC}}$. The simulation results showed that when the input voltage changes the full-bridge phase-shift ZVS power converter at the rated voltage of $45 \mathrm{~V}-50 \mathrm{~V}$, the output voltage of the full-bridge phase-shift ZVS power converter is able to control the output voltage at constant $400 \mathrm{VDC}$.

\section{References}

[1] S. K. Mazumder, R. Burra, R. Huang, M. Tahir, K. Acharya, G. Garcia, S. Pro, O. Rodrigues, and E.Duheric, "A universal grid-connected fuel-cell inverter for residential application," IEEE Trans. Industrial Electronics, accepted and in press for publication, 2010.

[2] S. K.Mazumder, R. K. Burra, R. Huang, and V. Arguelles, "A low-cost single-stage isolated differential CCCuk inverter for fuel-cell application," in IEEE Power Electronics Specialists Conference, Rhodes, Greece, pp. 4426-4431, 2008.

[3] S. K. Mazumder, R. K. Burra, and K. Acharya, "Power conditioning system for energy sources," USPTO Patent\# 7,372,709 B2, awarded in May 13, 2008.

[4] Lai, C.; Yang, M.; Liang, S. A Zero Input Current Ripple ZVS/ZCS Boost Converter with BoundaryModeControl. Energies 2014, 7, 6765-6782. 
[5] Zhao, L.; Li, H.; Yu, Y.;Wang, Y. A Novel Choice Procedure of Magnetic Component Values for Phase Shifted Full Bridge Converters with a Variable Dead-Time Control Method. Energies 2015, 8, 9655-9669

[6] Liu, K.B.; Liu, C.Y.; Liu, Y.H.; Chien, Y.C.; Wang, B.S.; Wong, Y.S., “Analysis and Controller Design of aUniversal Bidirectional DC-DC Converter,". Energies 2016.

[7] Jauch, F.; Biela, J., "Combined Phase Shift and Frequency Modulation of a Dual Active Bridge AC-DC Converter with PFC," IEEE Trans. Power Electron., 31, 8387-8397, 2016.

[8] Hu Xuezhi; Nan Guangqun, "The Research of Modeling and Simulation for Phase-shifted Full-bridge ZVS DC / DC Converter," Third International Symposium on Intelligent Information Technology Application, 2009

[9] J.A. Sabate, V. Vlatkovic, R. B. Ridley, F. C. Lee, and B. H. Cho, "Small-signal analysis of zero-voltage switched full-bridge PWM converter", High Frequency Power Conversion Conf. '90 Rec., 1990, pp.262-272.

[10] N. Mohan, T. M. Undeland, W. P. Robbins, Power Electronics Converters, Application and Design, 3rd edition, Wiley-India, New Delhi, 2003.

[11] B.-R. Lin, K. Huang, and D. Wang, "Analysis and implementation of full-bridge converter with current doubler rectifier," IEE Proceedings Electric Power Applications, vol. 152, pp. 1193-1202, 2005.

[12] J. Sabate, V. Vlatkovic, R. Ridley, F. Lee, and B. Cho, "Design considerations for high-voltage high-power full-bridge zero-voltage switched PWM converter," in Proc. IEEE APEC, 1990, pp. 275-284.

[13] M. K. Kazimierczuk, Pulse-width modulated DC-DC power converters: John Wiley \& Sons, 2008.

[14] G.-B. Koo, G.-W. Moon, and M.-J. Youn, "Analysis and design of phase shift full bridge converter with series-connected two transformers," Power Electronics, IEEE Transactions on, vol. 19, pp. 411-419, 2004.

[15] B.-R. Lin, K. Huang, and D. Wang, "Analysis and implementation of full-bridge converter with current doubler rectifier," IEE Proceedings Electric Power Applications, vol. 152, pp. 1193-1202, 2005.

[16] J. Zhang Z. Ouyang, M. C. Duffy, M. E. Andersen, and W. G. Hurley, “Leakage Inductance Calculation for Planar Transformer with a Magnetic Shunt", 2013 IEEE Energy Convers. Congr. Expo. ECCE 2013, vol. 50, no. 6, pp. 643-648, 2013.

[17] H. R. Karampoorian, G. Papi, A. Vahedi, and A. Zadehgol, “Optimum Design of High Frequency Transformer for Compact and Light Weight Switch Mode Power suppy (SMPS)", 2006 IEEE GCC Conf., vol. 2, no. 5, pp. 1-6, 2006.

[18] J. Sabate, V. Vlatkovic, R. Ridley, F. Lee, and B. Cho, "Design considerations for high-voltage high-power full-bridge zero-voltage switched PWM converter," in Proc. IEEE APEC, 1990, pp. 275-284.

[19] M. K. Kazimierczuk, Pulse-width modulated DC-DC power converters: John Wiley \& Sons, 2008.

[20] Jain, A.K.; Ayyanar, R. PWM control of dual active bridge; Comprehensive analysis and experimental verification. IEEE Trans. Power Electron. 2011, 26, 1215-1227.

[21] Jung, J-.H.; Kim, H-.S.; Ryu, M-.H.; Baek, J-.W., "Design Methodology of Bidirectional CLLC Resonant Converter for High-Frequency Isolation of DC Distribution Systems," in Power Electronics, IEEE Transactions on, vol.28, no.4, pp.1741-1755, April 2013

[22] Xu. D.H, Zhao. C. H, and. Fan H. F“ A PWM plus phaseshift control bidirectional dc-dc converter, "IEEE Trans. Power Electron., vol. 19, no. 3, pp.666 - 675, 2004. 